\title{
Novel NKX2-5 germline mutation in a Moroccan child with transitional atrio-ventricular septal defect (tAVSD)
}

\author{
Ihssane El-Bouchikhi'1,2, Khadija Belhassan ${ }^{1}$, Fatima Zohra Moufid ${ }^{1,2}$, Mohammed Iraqui \\ Houssaini $^{2}$, Karim Ouldim ${ }^{1}$, Samir Atmani ${ }^{3}$ \\ ${ }^{1}$ Medical Genetics and Oncogenetics Laboratory, ${ }^{3}$ Medico-Surgical Unit of Cardio-pediatrics, Department of Pediatrics, \\ Hassan II University Hospital, Fez, Morocco; ${ }^{2}$ Laboratory of Microbial Biotechnology, Faculty of Sciences and Techniques, \\ University of Sidi Mohammed Ben Abdellah, Fez, Morocco. E-mail: ihssane.elbouchikhi@usmba.ac.ma \\ Recevied: 30th March 2017, Accepted: 4th May 2017
}

SUMMARY: El-Bouchikhi I, Belhassan K, Moufid FZ, Houssaini MI, Ouldim $\mathrm{K}$, Atmani S. Novel NKX2-5 germline mutation in a Moroccan child with transitional atrio-ventricular septal defect (tAVSD). Turk J Pediatr 2017; 59: 610-613.

Atrioventricular septal defect is a complex congenital heart defects (CHD) with a prevalence of approximately $4 \%$ of all CHDs. Transitional form of atrio-ventricular septal defect (tAVSD) associates ostium primum atrial septal defect, common atrioventricular annulus with distinct atrioventricular valvar orifices in addition of restrictive ventricular septal defect. We describe in this report clinical and molecular features of a Moroccan boy that carries a novel NK2 homeobox 5 (NKX2-5) germline mutation (Pro141Ala), and exhibits a transitional atrio-ventricular septal defect. This phenotype has never been reported in association with NKX2-5 germline mutations. Pro141Ala is a non-reported pathogenic mutation that alters the nuclear localization signal sequence, leading to disruption of NKX2-5 nuclear translocation mechanism. Such alteration would decrease nuclear transcriptional activity of NKX2-5 and impair cardiogenesis process. The present report comes to widen the phenotypic spectrum of congenital heart disease caused by NKX2-5 germline mutations, and highlights as well the importance of the nuclear localization system in NKX2-5 activity.

Key words: atrio-ventricular septal defect (AVSD), NKX2-5, nuclear localization site (NLS)

Atrioventricular septal defect ([OMIM 606215; AVSD]) is one of the common complex congenital heart defects (CHDs) with an incidence around $4 \%$ of all CHDs observed at childhood. Atrioventricular septal defect (AVSD) is characterized by common atrioventricular annulus (with one or two AV valvar orifices), associated with large interatrial shunting at the primum septum, with or without interventricular communication. Depending on which of these conditions are combined, four AVSD types could be distinguished: complete (cAVSD), intermediate (iAVSD), transitional (tAVSD), and partial AVSD (pAVSD). ${ }^{1}$ Transitional form of AVSD associates primum atrial septal defect and common atrioventricular annulus with distinct atrioventricular valvar orifices in addition of restrictive ventricular septal inlet. ${ }^{2}$
Non-syndromic AVSD was demonstrated to be associated with pathogenic germline mutations in cardiac transcription factors such as GATA4 (GATA-binding protein 4) and TBX5 (T-Box5) ${ }^{3,4}$, and also with mutations in the cell adhesion molecule CRELD1 (cysteinerich protein with EGF-like domains 1). ${ }^{5}$ In contrast, AVSD cases caused by deficient NKX2-5, were rather associated with somatic mutations. Indeed, although the spectrum of CHDs caused by NKX2-5 germline mutations is quite large, comprising ASD with or without AV block, tetralogy of Fallot (TOF), ventricular septal defect (VSD), double-outlet right ventricle (DORV), interrupted aortic arch (IAA), hypoplastic left heart syndrome (HLHS) amongst others, no NKX2-5 germline missense mutations were detected to date in 


\section{AVSD patients. ${ }^{6-9}$}

We report in this paper a case of a Moroccan patient that carries a novel NKX2-5 germline mutation, and exhibits a transitional atrioventricular septal defect (tAVSD), a phenotype usually associated with NKX2-5 somatic mutations.

\section{Case Report}

The patient is a 2-year-old boy, born to healthy, non-consanguineous parents from the central North Morocco. The father and the mother were respectively 38 and 26 years old. In the neonatal period, he suffered from jaundice and recurrent respiratory infections. At two months of age, he was admitted to pediatric hospital due to afebrile acute dry cough associated with dyspnea and cyanosis of the lips and fingertips. At the admission, his weight and height was respectively $4.5 \mathrm{~kg}(-3 \mathrm{SD})$ and $54 \mathrm{~cm}(-3$ $\mathrm{SD})$. At auscultation, the infant showed systolic heart murmur and tachycardia with pulse reaching 160/min and respiratory rate of 60/ min. Echocardiography revealed a transitional AVSD consisting of a large ostium primum atrial septal defect with left-to-right shunting and restrictive ventricular septal defect (Fig. 1).

\section{Molecular analysis}

The proband was assigned to genetics laboratory for molecular testing. After obtaining the informed consent as well as the approval of the local ethic committee (under the report number Réf.06/14), the blood sample was taken and genomic DNA was extracted. Then, we

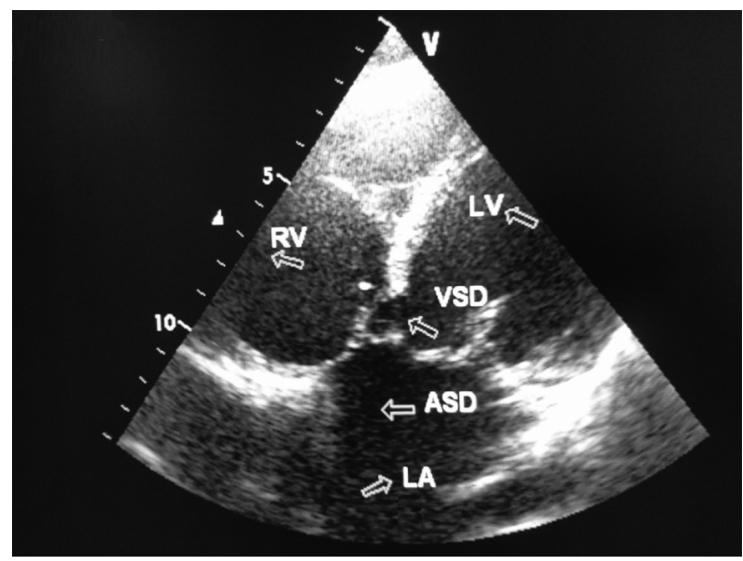

Fig. 1. Echocardiogram of the proband showing a tAVSD phenotype with a large ostium primum atrial septal defect and a restrictive ventricular septal defect. carried out polymerase chain reaction (PCR) amplification of the two NKX2-5 coding exons and their respective boundaries according to the standard protocol. The PCR product was directly sequenced using 3500Dx Genetic Analyser (Applied Biosystems, Massachusetts, MA, USA). The Bioinformatics analysis of the obtained sequences revealed a $\mathrm{C}$ to $\mathrm{G}$ transition at position 421 (c. $421 \mathrm{C}>\mathrm{G})$. This missense mutation located in the exon 2 leads to amino acid substitution p.Pro141Ala within the NKX25 homeodomain (HD) (Fig. 2).

\section{Discussion}

NKX2-5 is a member of NKX2 transcription factor family. This highly conserved homeobox gene is widely expressed in cardiac cells throughout the heart development. NKX2-5 interacts with GATA4 and TBX5 to regulate the downstream genes involved in several cardiogenesis processes. ${ }^{10}$

Germline mutations affecting this transcription factor (NKX2-5) were proved to cause a wide range of congenital heart defects, concerning mainly ASD, TOF, and VSD. ${ }^{10,11}$ The current literature confirms that NKX2-5 mutations identified in AVSD subjects were rather reported in a somatic context. ${ }^{6-9}$ Thus, the present article reports the novel NKX2-5 germline mutation Pro141Ala that was found, for the first time in association with transitional atrio-ventricular septal defect (tAVSD).
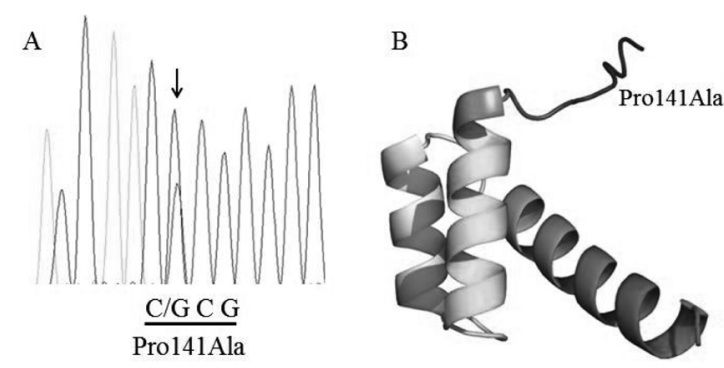

C

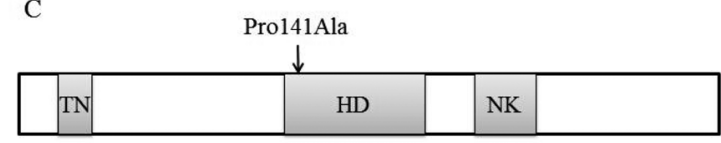

NKX2-5

Fig. 2. Sequencing chromatogram of the detected NKX2.5mutation c. 421C $>$ G A) Three-dimensional (3D) model of NKX2-5 homeodomain according to Phyre2, B) Organization of NKX2.5 domains with the detected mutation site; C) HD: homeodomain, TN: Tinman domain. 


\begin{tabular}{|c|c|c|}
\hline apiens & 41 & 2VYELERRFKQQRYLSAPERDQLASVLKLTSTQVKIWEQN \\
\hline P.troglodytes & 141 & RLFSQAQVYELERRFKQQRYLSAPERDQLASVLKLTSTQVKIWFQNRR \\
\hline M.mulatta & 141 & VYELERRFKQQRYLSAPERDQLASVLKLTSTQVKIWEQNRR \\
\hline pus & 40 & RVLFSQAQVYELERRFKQQRYLSAPERDQLASVLKLTSTQVKIWFQNRR \\
\hline B.ta & 140 & VLFSQAQVYELERRFKQQRYLSAPERDQLASVLKLTSTQVKIWFQNRR \\
\hline culus & 40 & RVLFSQAQVYELERRFKQQRYLSAPERDQLASVLKLTSTQVKIWFQNRR \\
\hline R.norvegicus & 140 & RVLFSQAQVYELERRFKQQRYLSPAERDQLLASVLKLTSTQVKIWFQNRR \\
\hline G.gallus & 122 & RVLFSQAQVYELERRFKQQKYLSAPERDHLANVLKLTSTQVKIWFQNRR \\
\hline & 44 & VLFSQAQVYELERRFKQQKYLSAPERDHLANVLKLTSTQVKIW \\
\hline X.tropicalis & 128 & VLFSQAQVYELERRFKQQKYLSAPERDHLANVLKLTSTQVKIWEQNRR \\
\hline
\end{tabular}

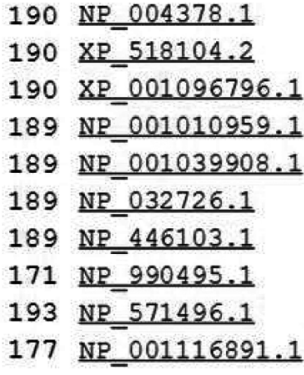

Fig. 3. Multiple alignment of NKX2-5 protein sequences across species showing the Prol41 residue conservation among species.

Missense mutation c. $421 \mathrm{C}>\mathrm{G}$, that causes the Pro141Ala amino acid change, is localized in the amino terminus of NKX2-5 DNA-binding homeodomain (HD). This 60-amino-acids domain plays a critical role in transactivation of the down-stream target genes through binding to their 5'TC/TAAGTG consensus binding sites. ${ }^{12}$ Multiple alignments of NKX25 protein sequences across species exhibit the strong conservation of this residue through the different species (Figure 3), which suggests an important role in the homeodomain function.

Disease-causing potential of Pro141Ala mutation assessed through different functional consequence prediction tools shows that this mutation is predicted to have a disease-causing effect according to Mutation Taster, probablydamaging effect according to PolyPhen, and deleterious effect on protein function according to SIFT prediction tool.

The pathogenicity of this mutation could be explained by the particular importance of codon Pro141. Indeed, this residue lies particularly within the NKX2-5 nuclear localization signal (NLS) sequence, which is a short consensus sequence at the homeobox amino terminus that is crucial for transporting the protein to the nuclear pore. Therefore, mutations affecting this sequence disrupt the nuclear translocation mechanism, and thus reduce the NKX2-5 transcriptional activity. 13

Pro141Ala is a novel mutation that was not reported in any published work. Moreover, this pathogenic mutation is not mentioned in the well-known human mutation databases, such as NCBI SNP database (dbSNP) at http:// www.ncbi.nlm.nih.gov/snp, 1000 genomes at http://browser.1000genomes.org, Exome
Aggregation Consortium (ExAC) at http://exac. broadinstitute.org, Human Genome Database (HGMD) at http://www.hgmd.cf.ac.uk, and NHLBI GO Exome Sequencing Project (ESP) at http://evs.gs.washington.edu/EVS.

Taken together, these findings suggest that Pro141Ala is a novel pathogenic mutation that seems to be responsible of the transitional AVSD observed in our proband.

It would have been more interesting if we had screened the parents and siblings' DNA to find out whether the mutation is sporadic or familial, as well as its segregation mode, but unfortunately, parental and sibling DNA samples were not available.

In conclusion, our report emphasizes particularly the importance of nuclear localization system in NKX2-5 activity and provides further evidence that NKX2-5 homeodomain alteration lead to an aberrant cardiac development. On the other hand, the reported transitional AVSD case comes to widen the spectrum of congenital heart disorders caused by NKX2-5 germline mutations.

Finally, in order to deepen the understanding of the molecular mechanism through which the mutation causes the observed phenotype, further functional studies of Pro141Ala would be of great interest.

\section{Acknowledgments}

We thank the patient and his family for their participation in this study. This work was financially supported by Hassan II University Hospital and the Faculty of Medicine and Pharmacy of Fez - University of Sidi Mohamed Ben Abdellah, Morocco. 


\section{REFERENCES}

1. Cetta F, Minich L, Edwards W, Dearani JA, Puga FJ. Atrioventricular septal defects. In: Allen HD, Driscoll DJ, Shaddy RE, Feltes TF (ed). Moss and Adam's Heart Disease in Infants, Children, and Adolescents. Philadelphia: Lippincott Williams \& Wilkins, 2008: 646-666.

2. Nii M. Assessment of Atrioventricular valve anatomy and function in congenital heart diseases using threedimensional echocardiography. In: Senzaki H, Yasukochi S (ed). Congenital Heart Disease. Japan: Springer, 2015: 21-42.

3. Reamon-Buettner SM, Borlak J. GATA4 zinc finger mutations as a molecular rationale for septation defects of the human heart. J Med Genet 2004; 42: e32.

4. Reamon-Buettner SM, Borlak J. TBX5 mutations in non-Holt-Oram syndrome (HOS) malformed hearts. Hum Mutat 2004; 24: 104.

5. Robinson SW, Morris CD, Goldmuntz E, et al. Missense mutations in CRELD1 are associated with cardiac atrioventricular septal defects. Am J Hum Genet 2003; 72: 1047-1052.

6. Wessels M. and Willems P. Genetic factors in nonsyndromic congenital heart malformations. Clin Genet 2010; 78: 103-123.
7. Inga A, Reamon-Buettner SM, Borlak J, Resnick MA Functional dissection of sequence-specific NKX2-5 DNA binding domain mutations associated with human heart septation defects using a yeast-based system. Hum Mol Genet 2005; 14: 1965-1975.

8. Reamon-Buettner SM, Borlak J. Somatic NKX2-5 mutations as a novel mechanism of disease in complex congenital heart disease. J Med Genet 2004; 41: 684690.

9. Reamon-Buettner SM, Hecker H, Spanel-Borowski K, Craatz S, Kuenzel E, Borlak J. Novel NKX2-5 mutations in diseased heart tissues of patients with cardiac malformations. Am J Pathol 2004; 164: 2117-2125.

10. Benson DW, Silberbach GM, Kavanaugh-McHugh A, et al. Mutations in the cardiac transcription factor NKX2-5 affect diverse cardiac developmental pathways. J Clin Invest 1999; 104: 1567-1573.

11. Elliott DA, Kirk EP, Yeoh T, et al. Cardiac homeobox gene NKX2-5 mutations and congenital heart disease. J Am Coll Cardiol 2003; 41: 2072-2076.

12. Chen CY, Schwartz RJ. Identification of novel DNA binding targets and regulatory domains of a murine tinman homeodomain factor, nkx-2.5. J Biol Chem 1995; 270: 15628-15633.

13. Kasahara $\mathrm{H}$, Izumo S. Identification of the in vivo casein kinase II phosphorylation site within the homeodomain of the cardiac tissue-specifying homeobox gene product Csx/Nkx2.5. Mol Cell Biol 1999; 19: 526-536. 